\title{
Le concept de risque perçu en psychologie du consommateur : antécédents et statut théorique
}

\author{
Article publié dans la revue Recherche et Applications en Marketing, \\ par Pierre Volle (ESSEC et Université Paris Dauphine), en 1995
}

\section{INTRODUCTION}

D'après Bauer (1960), le comportement du consommateur implique un risque dans le sens où chaque achat va engendrer des conséquences, parfois négatives, qu'il ne peut anticiper avec certitude. Depuis cette contribution fondatrice, l'étude du risque perçu se révèle l'une des traditions de recherche les plus fécondes et les plus dynamiques de la littérature en comportement du consommateur. Depuis plus de trente ans, le foisonnement des recherches allant de pair avec une complexité croissante, un bilan de ces différents travaux est justifié, tant au plan théorique qu'au plan pratique.

Au plan théorique, alors qu'un vaste courant de recherches s'est développé depuis le milieu des années soixante (Cox, 1967b), les synthèses disponibles (Ross, 1975; Stem, Lamb et Maclachlan, 1977) ne prennent pas en compte les travaux plus récents, comme ceux qui remettent partiellement en cause la théorie de l’utilité espérée sur laquelle repose le risque perçu (Kahneman et Tversky, 1979; Bell, Raiffa et Tversky, 1989). De plus, ce qui n’est pas le cas en théorie de la décision, le concept de risque perçu comme théorie centrale reste à construire en psychologie du consommateur (Ingene et Hughes, 1985).

Au plan pratique, le risque perçu se révèle un critère de segmentation (Peter et Ryan, 1976) ou de ciblage des stratégies de réduction du risque sur des segments particuliers (Stem et Lamb, 1980; McCorkle, 1990). 
Par ailleurs, la prise en compte du risque perçu améliore la performance des entreprises par une meilleure adaptation de leur mix, qu'il s’agisse, par exemple, du conditionnement, de la communication, du canal de distribution ou des attributs du produit, comme le prix ou la sécurité (Havlena et DeSarbo, 1991). Les applications disponibles illustrent l'attrait du risque pour comprendre et influencer les processus de choix des biens de consommation courante, comme les vins (Mitchell et Greatorex, 1989), les céréales du petit déjeuner (Mitchell et Boustani, 1993) ou les biens durables comme les vêtements (Lumpkin, Crawford et Kim, 1985), les ordinateurs (Mitchell, 1990) et les voitures d'occasion (Gabbott, 1991).

Afin de limiter la taille de l'article, compte tenu de cette longue tradition de recherche, il a été nécessaire de poser trois limites. Tout d'abord, le risque perçu par les acheteurs industriels n’est pas traité (e.g. Sheth, 1973; Newall, 1977; Pras et Tarondeau 1981; Puto, Patton et King, 1985; Henthorne, LaTour et Williams, 1993). De plus, seules sont prises en compte les décisions individuelles, laissant de côté les décisions en groupe dont une revue de la littérature pourrait justifier un article à elle seule (e.g. Kogan et Wallach, 1964; Woodside, 1972; Davis et al., 1992). Une dernière limite réside dans le choix de se situer au sein du courant de la psychologie descriptive - celle qui s’intéresse à la manière dont les individus et en particulier les consommateurs évaluent réellement le risque - par opposition à la psychologie normative qui suppose souvent une rationalité parfaite du consommateur (distinction très classique présentée en détail par les auteurs suivants : Abelson et Levi, 1985; Bell, Raiffa et Tversky, 1988; Hogarth, 1989).

Comme le montre la figure 1, une structure tripartite s'impose d'elle-même : le concept de risque perçu et ses méthodes de mesure sont d’abord exposés (I); les antécédents du risque perçu en psychologie du consommateur sont présentés (II), avant que la question du statut théorique de la variable de risque ne soit soulevée (III).

< insérer la figure $1>$ 


\section{LE CONCEPT DE RISQUE PERÇU ET SES METHODES DE MESURE}

Les composantes du risque perçu, comme le concept de risque global, font l'objet d'une première analyse. Les méthodes de mesure du risque perçu sont ensuite exposées.

\section{LES COMPOSANTES DU RISQUE ET LE RISQUE GLOBAL}

Le risque consiste en la perception d'une incertitude relative aux conséquences négatives potentiellement associées à une alternative de choix. Le risque est donc la possibilité de subir des pertes à l'occasion de l'achat ou de la consommation d'un produit, bien ou service. Malgré sa simplicité, cette définition recouvre souvent des notions différentes d'un auteur à l'autre.

Les deux composantes du risque - pertes et incertitude - seront analysées, avant de s’attacher à la notion de risque global. Une définition du risque perçu en psychologie du consommateur sera finalement proposée.

\section{Les composantes du risque perçu}

La composante de perte sera précisée, avant de souligner sa nature multi-dimensionnelle. La composante d’incertitude sera ensuite présentée.

<insérer la figure 2> 
La composante de perte

Dans les premiers travaux (Cox, 1967d), les pertes potentielles comprennent : les buts visés mais non atteints; les pénalités éventuelles infligées au consommateur par son environnement; la perte des moyens engagés dans l'achat, comme l'argent ou le temps; enfin, tout autre "danger" associé à l'achat. D'autres auteurs (Peter et Tarpey, 1975; Humphreys et Kenderline, 1984) s'intéressent au résultat net attendu, c'est-à-dire au solde entre les conséquences positives et les conséquences négatives. La maximisation de ce solde explique plus de variance quant à la préférence entre les marques, que la maximisation partielle des gains ou des pertes (Peter et Tarpey, 1975).

La notion de perte qui sera retenue ici provient plutôt de la littérature en psychologie et caractérise la situation où un individu obtient un résultat inférieur à un point de référence (Kahneman et Tversky, 1979; Yates, 1992). Par exemple, pour une voiture, 150000 km sans changer le moteur sera le point de référence pour tel individu; tout modèle qui ne réalisera pas cette distance avec le même moteur engendrera la perception d'une perte dont l'ampleur variera en fonction de l'écart entre le résultat obtenu et le point de référence.

Le point de référence peut apparaître de multiples façons (Yates et Stone, 1992a) : une expérience personnelle, une référence sociale, une valeur-cible à atteindre, le meilleur résultat possible ou encore le regret maximum par rapport aux autres alternatives.

\section{La composante d'incertitude}

L'incertitude peut porter sur de nombreuses étapes du processus d'évaluation des pertes attachées aux alternatives de choix (Yates et Stone, 1992b). Un exemple portant sur l'achat d'une voiture d'entrée de gamme, permettra de mieux en cerner les contours.

<insérer le tableau 1> 
Le plus souvent, l'ensemble des alternatives offertes au choix n'est pas connu avant une recherche d'information intensive, et même après. L'estimation des attributs soulève également de nombreuses incertitudes : quels sont les attributs déterminants pour le choix et comment les alternatives se positionnent-elles pour chaque attribut ? L'estimation des points de référence, comme l'évaluation des attributs, constituent aussi des étapes incertaines. La question centrale devient alors : quelle est la valeur d'un niveau donné d'attribut, en particulier dans le cas de produits nouveaux pour l'acheteur?

Concernant la nature de l'incertitude, la plupart des situations d'achat sont dite "ambiguës" car elles sont intermédiaires entre l'incertitude totale - les pertes potentielles sont ignorées et l'incertitude objective - une probabilité connue est attachée à chaque perte potentielle (Einhorn et Hogarth, 1986). En psychologie du consommateur, on parlera de probabilités subjectives, pour traduire la difficulté à estimer précisément les degrés d’incertitude dans la réalité.

L’incertitude affecte le processus de décision - plus la situation est incertaine quant aux alternatives à choisir, plus la recherche d'information est active (Urbany, Dickson et Wilkie, 1989 - mais aussi le poids des attributs sur lesquels elle porte - l’importance d'un attribut qui améliore l'utilité est d'autant plus élevée que son niveau d’incertitude est bas (Kahn et Meyer, 1991) et inversement pour un attribut de valence négative.

\section{Le risque global}

La décomposition du risque en deux composantes est avant tout heuristique. En fait, les questions de recherche essentielles se posent plutôt par rapport au risque global : quelles en sont les dimensions (quelles sont les pertes effectivement prises en compte par le consommateur dans son choix), quel en est le niveau de perception (le consommateur perçoit-il un risque global, ou au contraire un risque finement décomposé selon les dimensions). 
Finalement, la perception du risque par un individu suit-elle les axiomes de la théorie de l'utilité espérée (quel est le degré de rationalité qui caractérise les individus lorsqu'ils évaluent le risque) ?

\section{Les dimensions du risque global}

Les pertes suivantes, appelées "dimensions du risque global", ont été identifiées : le risque financier, le risque fonctionnel, le risque physique, le risque social, le risque psychologique (Jacoby et Kaplan, 1972; Kaplan, Szybillo et Jacoby, 1974), le risque de perte de temps (Roselius, 1971) et le risque d'opportunité (Zikmund et Scott, 1973), qui recouvre la perte associée à un achat dont le rapport qualité / prix évolue rapidement (e.g. l'électronique). Il a été montré que le risque global peut être appréhendé d'une manière satisfaisante à travers une ou deux dimensions principales : le risque fonctionnel, c'est-à-dire la performance, pour Jacoby et Kaplan (1972) ou alors le risque financier et le risque psychologique pour Stone et Grönhaug (1993). Les travaux de Derbaix (1983) permettent de penser que cette dimension principale dépend du type de produit étudié.

Plus récente, la conception multi-attributs est venue enrichir notablement le concept de dimension du risque. Dans ce cadre d'analyse, il existe autant de dimensions que d'attributs déterminants pour un produit donné, dans une situation d'achat donnée, pour un individu donné (ou un segment d'individus); la perte peut s'attacher autant au produit, qu'aux services associés : garantie (Vann, 1987), service après-vente, maintenance... L'incertitude apparaît dès le modèle de Fishbein (1967), même si la distinction entre "quantité de l'attribut $i$ dans l'objet $o$ " et "probabilité que l'attribut $i$ se trouve dans l'objet $o$ " n'est pas suggérée. Le modèle d'Athola (1973) introduit explicitement la notion de distribution de probabilité sur les niveaux d'attributs comme composante de son modèle. Finalement, dans les travaux de Möller (1983), de nombreuses dimensions sont appréhendées : présence et niveau de chaque attribut, capacité de chaque niveau d'attribut à satisfaire un niveau de but. Ce modèle prend en compte à la fois les situations risquées pour lesquelles les probabilités sont connues et les situations incertaines pour lesquelles une variance est attachée à la distribution de probabilités (distinction classique introduite par Knight, 1921). 
L'approche multi-attributs, qui sera retenue pour la définition, est certainement plus pertinente que l'approche à partir des grandes dimensions; en effet, aucune décomposition du risque global ne s'impose arbitrairement. De plus, rien n'empêche de réduire le nombre élevé d’attributs à quelques axes pertinents (Havlena et DeSarbo, 1991).

<insérer le tableau 2>

\section{Les niveaux de perception du risque global}

La question s'est posée de savoir si le risque est perçu par le consommateur d'une manière globale - au niveau de chaque situation d'achat ou de chaque alternative - ou au contraire, si le risque est perçu d’une manière décomposée - au niveau de chaque dimension.

Vann (1983) a proposé un modèle conceptuel de composition du risque par strates, où plusieurs distributions de probabilités partielles s'emboîtent pour construire progressivement le risque global. Le débat commence à se développer en théorie de la décision (Yates et Stone, 1992b) et il semble surtout que les représentations du risque dépendent des situations de choix.

La conception multi-attribut suppose que le consommateur perçoive le risque au niveau de chaque attribut déterminant, ce qui peut se révéler irréaliste dans certaines situations de choix. Plus spécifiquement, pour les situations où le temps est limité, la représentation du risque serait plus simple, le risque s'attachant à chaque alternative et non pas aux différentes dimensions de ces alternatives (Yates, 1992).

Le risque global et la théorie de l'utilité espérée

Des modifications axiomatiques de la théorie de l’utilité espérée (Von Neumann et Morgenstern, 1947) ont été proposées afin d’intégrer les travaux empiriques qui modèrent l'hypothèse fondamentale selon laquelle le consommateur choisit entre plusieurs alternatives sur la base d'une maximisation de l'utilité attendue d'un ensemble de résultats, 
pondérés par leur probabilité d'occurrence. Ces travaux empiriques appartiennent à trois champs de recherche : la théorie des prospects, les biais cognitifs et les heuristiques.

La théorie des prospects (Kahneman et Tversky, 1979; Tversky et Kahneman, 1992) montre que l'appréhension des situations de choix risqués est caractérisée par des biais importants quant au codage des alternatives durant la phase de simplification du choix, d'évaluation des alternatives (traitement différent des pertes et des gains) et d'estimation des probabilités (surestimation des petites probabilités...). Dès lors, l'attitude face au risque dépend étroitement de la manière dont la situation est présentée; ainsi, il est montré que les individus craignent le risque pour les situations de gain et recherchent le risque pour les situations de perte.

De nombreux biais cognitifs ont également été identifiés : non indépendance de l'évaluation des pertes et des probabilités (Kahn et Meyer, 1991); prévisions ancrées sur les scénarii les plus disponibles, c'est-à-dire les plus optimistes (Kahneman et Lovallo, 1993); conservatisme pour réviser les probabilités; négligence à considérer des hypothèses alternatives... Plusieurs travaux de synthèse permettent de mieux appréhender la perception du risque en psychologie descriptive (Hogarth, 1986; Fischhoff, 1992).

La mise en œuvre d'heuristiques - tactiques destinées à simplifier les processus d'évaluation et de choix - conduit également à déformer la perception des événements incertains (Tversky et Kahneman, 1982). Par exemple, l'heuristique de disponibilité, qui a fait l'objet de recherches dans le domaine de l'attribution des défaillances de produits (Folkes, 1984), pousse les individus à considérer comme plus probables les événements qui viennent à l'esprit le plus facilement, sans lien nécessaire avec leur probabilité réelle. Ainsi, le nombre de suicides sera sous-estimé alors que le nombre de morts en avion, plus médiatisé, sera systématiquement surestimé (Folkes, 1988). 
L'ensemble de ces travaux souligne la subjectivité qui préside à la perception des composantes du risque, clé d’une définition qui serait sans doute assez largement partagée par la communauté des chercheurs. Le risque est l'incertitude subjectivement perçue par un individu quant à l'ensemble des pertes potentielles relatives aux attributs déterminants du choix d'un produit (bien ou service) dans une situation d'achat ou de consommation donnée; une perte s'entend comme un résultat qui se révélerait inférieur à un point de référence subjectif, qui n’est pas nécessairement le zéro ou le statu quo, mais qui peut être le niveau atteint par la meilleure alternative ou encore toute autre référence propre à l’individu.

On notera que le concept de satisfaction peut être rapproché du concept de risque ${ }^{1}$. Il est vrai que la littérature sur la satisfaction (e.g. modèle de non confirmation des attentes de Oliver) présente un large point commun avec celle du risque : l'analyse en composantes multi-attributs (Oliver, 1980; Tse et Wilton, 1988). Néanmoins, pour le risque, l'estimation de la différence entre points de référence et résultats précède l'achat; c’est donc sur la composante d'incertitude que repose la spécificité du concept de risque par rapport à celui de satisfaction. On notera également que dans la littérature, seul l'aspect essentiellement cognitif du risque est traité, ce que l'on peut regretter. En fait, dans le cadre de cette théorie du risque perçu, la dimension affective est entièrement captée par les émotions, au premier rang desquelles la peur et l’anxiété (Taylor, 1974), qui sont des conséquences du risque et non des composantes.

La définition proposée ci-dessus s’articule explicitement sur la décomposition du risque en deux composantes et les méthodes d'opérationnalisation du risque, objet de la partie qui suit, s’y adossent en grande partie.

\footnotetext{
${ }^{1}$ L'auteur remercie deux lecteurs anonymes pour cette suggestion.
} 


\section{LES METHODES DE MESURE DU RISQUE}

\section{Les méthodes déclaratives directes}

Parfois remise en cause (Gemünden, 1985), la validité des méthodes directes de mesure a été établie par Lumpkin et Massey (1983). Les deux composantes sont estimées séparément par des questions directes puis combinées d'une manière multiplicative (Cunningham, 1967a), parfois additive (Bettman, 1973), ou maintenues distinctes avant analyse factorielle complémentaire (Laurent et Kapferer, 1986). La méthode de Cunningham (1967a), la plus fréquemment utilisée en marketing (e.g. Dash et al., 1976; Güseman, 1981; Verhage et al., 1990), permet d’illustrer le principe de combinaison des deux composantes, notées sur une échelle de 1 à 4 selon le degré d’incertitude et de "danger". A partir de cette méthode, un indice de 16 indique un risque faible alors qu'un indice de 1 indique un risque fort.

<insérer le tableau 3>

Un dernier ensemble de méthodes directes consiste à obtenir une estimation du risque global, sans décomposition, à partir d'une échelle ordinale ou cardinale (e.g. Roselius, 1971; Müller, 1985; Lumpkin et Dunn, 1990).

\section{Les méthodes indirectes}

Issues des travaux de Markovitz, les méthodes qui reposent sur la notion de variance sont caractéristiques des modèles multi-attributs (Möller, 1983). Pras et Summers (1978) montrent que des alternatives d'espérances mathématiques identiques quant aux attributs importants ne sont pas équivalentes au regard du risque. Les auteurs proposent alors de retenir l'écart type des résultats au dessous de la moyenne (semi standard deviation) pour rendre compte de la dispersion des valeurs et de la forme des courbes. 
Sous réserve d'accepter la validité des axiomes de la théorie de l'utilité, une méthode de mesure rigoureuse repose sur l'estimation d'une courbe d'utilité individuelle à partir de loteries au cours desquelles l'individu choisit entre des tickets dont les valeurs et les probabilités varient d’une manière systématique (Hauser et Urban, 1979; Currim et Sarin, 1983). Par exemple, si un individu préfère gagner $100 \mathrm{~F}$ plutôt que de jouer à la loterie "une chance sur deux de gagner 300 F sinon rien", il révèle une attitude négative face au risque puisque l'utilité de la loterie $(300 \mathrm{~F} * 50 \%=150 \mathrm{~F})$ est supérieure à celle du gain certain (100 F). Complexe, cette procédure est peu utilisée en marketing. Toutefois, il est possible de l'adapter, tout en conservant le principe d'un choix entre des alternatives incertaines (Roux, 1991).

D'autres auteurs (Havlena et DeSarbo, 1991) utilisent une forme d'analyse conjointe pour déterminer l'impact d'une modification des éléments du mix sur le risque perçu au niveau individuel, sans mesurer le risque per se de chaque alternative, mais en comparant leurs niveaux relatifs.

\section{Les méthodes expérimentales}

Des procédures expérimentales de manipulation sont également envisageables. Dès lors, il ne s'agit pas de mesurer le risque perçu, mais d'assigner les individus à des groupes dont les niveaux de risque sont supposés différents, suite à une manipulation expérimentale. et d’observer si les comportements sont différents selon les groupes. La manipulation concerne généralement les informations sur les attributs, comme le prix ou la réputation du producteur (Ring et al., 1980). Dans une autre étude (Lantos, 1983), les pertes potentielles du groupe "risque fort" sont supérieures de 15 \% à celles du groupe "risque faible". 
Lorsque l'on choisit une méthode de mesure du risque, il s'agit avant tout d'éviter deux types d'erreurs qui apparaissent dans la littérature. Les mesures qui reposent sur des concepts dits équivalents, comme l'acceptation plus ou moins large des erreurs de type I et de type II (Barach, 1969; Schiffman, 1972) sont tout d'abord à exclure. En effet, ces méthodes reposent sur un lien a priori entre le risque et le concept dit "équivalent". De même, il s'agit de ne pas confondre la mesure du concept de risque perçu avec ses manifestations supposées (e.g. Locander et Hermann, 1979); Ross (1975) souligne le problème de la confusion entre le critère (les manifestations du risque) et le construit (le risque) : si les instruments mesurent autre chose que les manifestations du risque, on induit le risque alors qu'il n'existe pas. Ainsi, même si la méthode de Cunningham n’est pas exempte d'écueils - confusion entre "utilisation" du produit et "achat ou consommation", classement approximatif des indices (1 à 16) en trois degrés de risque, choix arbitraire d'une combinaison multiplicative linéaire des composantes - il peut être judicieux de l'utiliser pour faciliter les comparaisons entre recherches.

En guise de conclusion, ces méthodes de mesure devraient plus s’attacher à caractériser la nature du risque qu’à simplement mesurer son degré. Connaître les antécédents du risque permettrait ainsi de mesurer le concept d'une manière plus "qualitative", en suivant une démarche proche des travaux sur les profils d'implication (Laurent et Kapferer 1986). Dans cette perspective, la présentation des antécédents du risque, étudiés à travers de nombreuses recherches, fait l'objet de la partie qui suit.

\section{LES ANTÉCÉDENTS DU RISQUE PERÇU}

Les antécédents du risque sont multiples. D’après la littérature, les facteurs divers qui favorisent voire engendrent la perception d'un risque avant l'achat peuvent être liés à l’individu, au produit, mais aussi à la situation d'achat. 
<insérer la figure 3>

\section{Les antécédents liés à l’individu}

L’influence des variables psychographiques est contrastée. Les individus dont le style cognitif est caractérisé par un fort désir de clarification (Cox, 1967c), une importante capacité de différenciation ou une tolérance élevée à l'ambiguïté (Müller, 1985) perçoivent un risque plus faible que les autres; les résultats de Müller sont vérifiés pour les produits de risque fort. Il a également été montré que la confiance en soi généralisée, une disposition générale stable, n'est pas corrélée avec le risque perçu (Cunningham, 1967a); en revanche, la confiance en soi spécifique, c'est-à-dire la confiance pour accomplir une tâche particulière, est positivement corrélée avec le risque perçu (Schaninger, 1976) ou avec la recherche d'information (Locander et Hermann 1979). L'anxiété est corrélée positivement avec le niveau du risque perçu (Schaninger, 1976), sans l'être avec l'activité de recherche d'information (Locander et Hermann, 1979). Schaninger (1976) montre que les mesures de risque perçu sont corrélées positivement avec la prise de risque comme trait de personnalité, concept sans doute proche de l'attitude face au risque en théorie de l'utilité. Une corrélation positive entre implication durable et risque perçu est également identifiée (Venkatraman, 1989).

L'influence des variables socio-démographiques apparaît pour un niveau de risque social élevé (Prasad, 1975). Globalement, elle est sans doute faible (Cunningham, 1967b), même si aucune étude récente ne vient confirmer ces résultats.

L'influence d'une variable d'environnement, comme la culture, sur la perception du risque a été montrée (Hoover, Green et Saegert, 1978; Verhage, Yavas et Green, 1991).

Dans l'ensemble, il manque un cadre analytique qui permette de dépasser les simples descriptions. La théorie de la motivation (Scheinder et Lopes, 1986) selon laquelle les comportements face au risque sont motivés par la sécurité ou, au contraire, par le rendement, fait figure d'exception. 


\section{Les antécédents liés au produit}

Dès les premières études, il a été montré que le risque dépend du type de produit considéré (Cunningham, 1967b; Perry et Hamm, 1969; Jacoby et Kaplan, 1972; Derbaix, 1983). Le pays d'origine du produit a également un impact sur le risque. Ainsi, Alden, Hoyer et Crowley (1993) montrent qu'à un produit d'implication faible dont le pays d'origine est perçu comme risqué - en l'occurrence une pâte dentifrice provenant du Mexique - est associé un processus de décision qui repose sur les attributs spécifiques du produit et non sur les attributs de la catégorie.

Une recherche montre l'importance de la marque dans la genèse du risque (Dunn, Skelly et Murphy, 1986) : le risque de performance est plus élevé pour les marques génériques, alors que le risque financier est plus élevé pour les marques nationales. D'autres auteurs (Roselius, 1971; Ring, Shriber et Horton, 1980) mettent en évidence que choisir une marque reconnue est un moyen efficace de réduire le risque.

Le prix est une variable qui peut jouer dans les deux sens; à un prix élevé est associé un risque financier élevé mais le prix est également un indicateur de qualité susceptible de réduire le risque (Zeithaml, 1988; Gijsbrechts, 1993). Ce résultat est contrasté car, pour Roselius (1971), choisir un produit de prix élevé n’est pas une stratégie efficace de réduction du risque.

D’autres facteurs liés au produit, même plus périphériques, ne doivent pas être négligés. Proposer une garantie de remboursement en cas d'insatisfaction permet de diminuer le risque perçu (Derbaix, 1983), dans une plus grande proportion que proposer un essai ou un échantillon gratuit (Akaah et Korgaonkar, 1988); cette dernière technique est d'ailleurs assez inefficace pour réduire le risque (Shoemaker et Shoaf, 1975). Offrir une garantie après-vente est également un facteur qui permet de réduire sensiblement le risque (Vann, 1987).

Le risque global attaché à la classe de produit est significativement plus élevé pour les services que pour les biens (Güseman, 1981). D'après Murray et Schlacter (1990), il en va de même pour les composantes physiques et psychologiques. Ces auteurs montrent également 
que le risque financier et le risque de performance sont plus élevés pour les services, mais uniquement auprès des répondants qui en ont une connaissance faible.

\section{Les antécédents liés à la situation d’achat}

La manière dont la situation est présentée, le cadrage (framing), influence l'issue du choix (Tversky et Kahneman, 1986). Ainsi, les individus craignent le risque lorsque l'alternative est présentée comme un gain mais acceptent, ou même recherchent le risque, lorsque l'alternative est présentée comme une perte (Kahneman et Tversky, 1979). De même, les conséquences négatives futures d'une décision sont plus fortement sous-évaluées (discounted) que les conséquences positives futures Mowen (1991) : la situation est jugée moins risquée lorsque son dénouement est lointain.

L'humeur de l'individu au moment du choix a un impact sur la perception du risque. Les individus pour lesquels une humeur positive a été induite en laboratoire se comportent d'une manière plus risquée que le groupe de contrôle, pour les situations de risque faible; le résultat inverse est obtenu pour les situations de risque élevé (Isen et Patrick, 1983). Toutefois, ces

sujets "heureux" manifestent un comportement de prise de décision différent, plus sommaire et de moins bonne qualité que les sujets "tristes" (Forgas, 1989).

Le temps est également un facteur qui pèse sur la perception du risque. Un manque de temps conduit à attacher plus de poids aux informations négatives et à être donc plus prudent (Abelson et Levi, 1985); il semble que l'heuristique de choix employée conduise avant tout à éviter les erreurs. Le choix de loteries plus sûres sous la pression du temps est aussi confirmé par Ben Zur et Breznitz (1981). Mann (1992) propose une synthèse détaillée quant à l'impact des émotions et du temps sur le risque.

Certains modes d'achat sont perçus comme plus risqués que d'autres, particulièrement l'achat par téléphone (Cox et Rich, 1964), l'achat par correspondance (Spence, Engel et Blackwell, 1970; Festervand, Snyder et Tsalikis, 1986; McCorkle, 1990), l'achat en magasin d'exposition, chez un discounter (Lumpkin et Dunn, 1990), ou l'achat électronique à domicile (Korgaonkar et Moschis, 1989). 
Au-delà des facteurs mentionnés ci-dessus, d'autres variables situationnelles ont sans doute un effet déterminant : la définition de la tâche, le destinataire du produit - un cadeau, par exemple - ou l'activité des producteurs sur le point de vente, comme la promotion ou la publicité sur le lieu de vente. A ce jour, aucune étude n'aborde sérieusement l'impact de ces facteurs situationnels sur le risque.

D'avantage qu'une source unique qui reposerait uniquement sur l'individu, le produit ou la situation, il semble qu'une explication pertinente de l'origine du risque se trouve dans l'interaction entre les individus et les situations d'achat, pour un produit, et donc un degré d’implication donné (Mischel, 1977; Bromiley et Curley, 1992). C’est sans doute la compréhension approfondie de ces interactions qui pourrait placer le risque en position centrale dans une théorie de la psychologie du consommateur. Pour l'heure, il s'agit d’appréhender la place que tient réellement le concept de risque dans les schémas explicatifs du comportement d'achat, c'est-à-dire son statut théorique.

\section{LE STATUT THÉORIQUE DU RISQUE PERÇU}

Le risque n'est pris en compte dans les modèles globaux de comportement du consommateur (Howard et Sheth, 1969; Engel, Blackwell et Miniard, 1990) qu'à travers la notion de confiance en soi pour l'évaluation des alternatives. En revanche, le risque perçu est une variable intermédiaire explicite dans les modèles de traitement de l'information (Cox, 1967; Bettman, 1979). Son statut théorique “ traditionnel ” est double; le risque est une étape du processus d'achat (l'étape d'évaluation du risque attachée à la situation et aux alternatives) mais également une variable intermédiaire qui affecte la suite du processus de décision. Audelà de ce rôle traditionnel, la littérature confère aussi au risque plusieurs autres prolongements théoriques dont nous allons traiter. 


\section{Le rôle traditionnel du risque perçu}

Une hypothèse centrale veut que le risque perçu détermine l'activité de recherche d'information, principale stratégie pour le réduire à un niveau acceptable (Cox, 1967). De même, le risque est souvent retenu comme composante de l'implication (Laurent et Kapferer, 1986). Enfin, les situations conflictuelles sont associées au risque (Janis et Mann, 1977).

\section{La recherche d'information}

Dans la mesure où l'information est un moyen de réduire l'incertitude (Cox, 1967d), l'activité de recherche d'information est différente selon le degré et les dimensions du risque perçu. Les communications inter-personnelles sont utilisées par les consommateurs pour limiter le risque perçu (Roselius, 1971), comme le "bouche à oreille" (Arndt, 1967; Perry et Hamm, 1969) et le contact avec des leaders d'opinion (Woodside et Delozier, 1976). Les informations commerciales, comme la publicité, sont considérées comme des stratégies moyennement performantes pour réduire le risque (Cox, 1967d; Roselius, 1971); sans doute en raison d'un problème de crédibilité. Les sources d'information dites "objectives", comme les publications qui testent les produits (e.g. 50 millions de consommateurs, Consumer Report), seraient surtout utilisées pour réduire le risque physique (Roselius, 1971).

Malgré son rôle central dans l'émergence théorique du concept de risque perçu, l'hypothèse selon laquelle la perception d'un risque élevé engendrerait une recherche d'information plus importante fait l'objet d'interrogations. Gemünden (1985) montre ainsi que la moitié des études empiriques conduisent à rejeter l'existence d'une relation positive entre le risque et l'activité de recherche d'information. Des explications sont avancées comme : un degré de risque inférieur à un seuil minimal, le coût de l'information, la dissonance potentielle générée par des informations contradictoires, ou le manque de confiance accordée aux sources d’information proposées dans ces études. Il reste que même l’hypothèse centrale qui confère au risque perçu son statut théorique repose sur des bases fragiles. Néanmoins, cette étude illustre la nécessité d'établir que les relations entre le risque et ses antécédents ne sont pas nécessairement linéaires. 


\section{L'implication}

La contribution majeure de Laurent et Kapferer (1986) renforce le courant de littérature qui considère le risque perçu comme une source de l'implication (Chaffe et McLeod, 1973; Muncy et Hunt, 1984; McQuarrie et Munson, 1986). Toutefois, comme nous l'avons vu plus haut, si le risque est une conséquence de l'implication durable, c'est aussi une source de l'implication situationnelle (Venkatraman, 1989). D'autre part, Strazzieri (1992, 1994) construit une échelle de mesure de l'implication qui repose sur "la distinction radicale entre risque et implication" car l'importance des pertes est la seule dimension commune aux deux concepts; par ailleurs, d'après l'auteur, le risque est attaché épisodiquement à l'achat alors que l'implication est attachée durablement à l'objet de l'achat. Pour Dandouau et Bensa (1993), les deux construits concourent parallèlement à l'apparition du besoin d'information sur le point de vente, mais l'implication est opérationnalisée avec deux items de risque, essentiellement pour des raisons de compatibilité avec les travaux antérieurs.

\section{Les situations conflictuelles}

Avec les travaux de Janis et Mann (1977), repris dans le modèle conceptuel de Bettman (1979), le risque perçu est invoqué à un double niveau : comme source du conflit et comme conséquence de sa gestion. Une situation est conflictuelle si les deux options de base, ne pas réagir ou réagir, génèrent un risque. Devant une situation de conflit, trois réactions sont alors possibles : l'évitement (reporter le choix sur quelqu'un d'autre, par exemple), l'hyper-vigilance (réagir d'une manière impulsive) et la vigilance (peser le pour et le contre, puis réagir). Pour ces auteurs, seule une réaction hyper-vigilante génère, en retour, une prise de risque élevée.

\section{Les prolongements théoriques du risque perçu}

Les prolongements qui suivent découlent de la littérature en psychologie du consommateur. Cependant, malgré leur intérêt pour la compréhension du comportement d'achat, ils sont rarement associés au concept de risque, même si ce dernier pourrait y puiser son statut théorique de variable intermédiaire majeure. 


\section{L'évaluation d'un produit (l'attitude)}

Le rôle du risque, ou plus précisément le rôle de l'incertitude, dans la formation de l'attitude a été étudié dans le cadre des modèles multi-attributs (pour une synthèse Möller, 1983). Ces modèles reposent sur la conception sous-jacente selon laquelle, plus l'incertitude relative à un attribut de poids positif est faible, plus l'attitude envers l'alternative de choix est positive. A l'inverse, plus l'incertitude relative à un attribut de poids négatif est faible, plus l'attitude envers cette alternative est négative. Parmi un ensemble d'autres variables pertinentes, le niveau de risque perçu déterminerait donc l'attitude globale envers un produit. La connaissance du vecteur des incertitudes et du vecteur des poids pour l'ensemble des attributs permettrait alors de hiérarchiser les attitudes envers les différentes alternatives.

Enfin, il a été montré que les consommateurs utilisent un plus grand nombre de dimensions pour évaluer les produits dont le risque est élevé; ceci traduirait une plus grande "vigilance cognitive" (Pinson et al., 1984).

\section{Le choix d'un produit (la préférence)}

Bettman (1973) distingue le risque inhérent du risque assumé. La différence entre les deux formes repose sur l'introduction de l'information dans le processus de décision. Ainsi, le risque inhérent est lié à la classe de produit : il sera plus faible pour une brosse à dents que pour une voiture, par exemple. Le risque assumé est celui pris par le consommateur, une fois la recherche d'informations réalisée, lorsqu'il choisit une marque parmi un ensemble d'alternatives. L'auteur suppose que le risque potentiellement assumé pour chaque marque est inférieur ou supérieur au risque inhérent à la classe de produit, selon que l'information acquise engendre ou non un risque perçu supplémentaire.

Bettman ne précise pas si l'alternative retenue est celle dont le risque assumé est le plus faible, toutes choses égales par ailleurs. A notre connaissance, cette hypothèse n'a jamais été spécifiquement testée dans un cadre commercial. En psychologie du consommateur, nous savons seulement que l'adoption d'un nouveau produit est freinée par la perception d'un risque élevé (Popielarz, 1967; Schiffman, 1972; Rogers, 1982). 
Le choix répété d'un produit (la fidélité)

Le risque ne serait pas seulement déterminant pour orienter le premier achat d'un produit ou d’une marque. En effet, la fidélité à une marque est reconnue comme une stratégie efficace de réduction du risque (Derbaix, 1983) : plus le risque perçu au niveau de la classe de produit est élevé, plus la fidélité à une marque est importante (Arndt, 1967b; Cunningham, 1967b; Roselius, 1971). Toutefois, ceci semble avant tout vrai aux États-Unis puisque Verhage, Yavas et Green (1991) ne valident pas l’hypothèse selon laquelle la fidélité est une stratégie efficace de réduction du risque, dans aucun des autres pays de leur étude : Turquie, Arabie Saoudite, Thaïlande et Pays-Bas.

\section{Le choix d'un mode d'achat et d'un point de vente (l'attitude envers l'enseigne)}

Certains modes d'achat étant perçus comme plus risqués que d'autres, des auteurs avancent que le choix du point de vente pourrait en partie dépendre du degré de risque qui lui est associé (Prasad, 1975; Dash, Schiffman et Berenson, 1976; Korgaonkar et Moschis, 1989). Néanmoins, une étude montre qu'il n'y a pas de relation entre la répétition des achats dans un même point de vente et le risque (Hisrich, Dornoff et Kernan, 1972). La contradiction pourrait s'expliquer par un niveau de risque très faible dans cette dernière étude. Il est vrai que ces auteurs testent l'hypothèse selon laquelle le choix du distributeur est une stratégie de réduction du risque, non que le choix s'effectue réellement sur cette base. Des travaux complémentaires sont donc souhaitables.

\section{CONCLUSION}

Cette article de synthèse s’est attaché à présenter d'une manière systématique les travaux significatifs en psychologie descriptive du consommateur qui précisent le concept de risque perçu, proposent des méthodes de mesure, identifient les antécédents du risque perçu et permettent de donner au concept de risque un statut théorique important, comme variable intermédiaire au sein du processus d'achat individuel. 
De multiples de voies de recherche sont envisageables :

- l'étude du risque perçu comme variable intrinsèquement affective et non pas seulement cognitive ;

- la mise au point d'une méthode de mesure du risque par "profils" qui ne conduirait pas seulement à un index uni-dimensionnel mais à un construit flexible dont la représentation et la perception tiendrait compte des interactions entre des variables individuelles et des variables situationnelles ;

- une approche expérimentale systématique pour dépasser le stade de la corrélation entre les antécédents et le concept de risque, puis étudier l'impact de manipulations du risque réel conséquences et probabilités réelles - sur le risque perçu ;

- la prise en compte explicite de relations non linéaires et non monotones entre le risque et ses antécédents ;

- la validation de l'hypothèse selon laquelle, toutes choses étant égales par ailleurs, le produit (ou le canal de distribution) de risque assumé le plus faible est systématiquement préféré par ceux qui ont une attitude négative face au risque ;

- la distinction entre le risque de l'échange et le risque du produit (Thaler, 1985); le risque de l'échange serait lié au mode d'achat, au point de vente, aux conditions de vente, etc. alors que le risque du produit, principalement évoqué dans ce document, serait le risque attaché à l'objet même de l'achat (cette analyse est d'autant plus pertinente pour les services pour lesquelles l'échange constitue le produit ou lorsque consommation et échange sont simultanés);

- la découverte des "représentations spontanées du risque que les individus construisent et utilisent dans leurs processus de décision " (Yates, 1992). Comme le montrent déjà quelques résultats, cette dernière voie est particulièrement prometteuse. Ainsi, Peter et Ryan (1976) mettent en évidence que la notion de perte est particulièrement pertinente pour le risque inhérent, alors que celle de probabilité l'est plus pour le risque assumé. En montrant que les individus fondent leurs heuristiques de choix sur les probabilités ou sur les conséquences selon leur niveaux respectifs, Diamond (1988) illustre également que cette voie est fructueuse. De même, Hansen (1992) montre empiriquement que les consommateurs naïfs 
concentrent leur attention sur les pertes, en considérant peu les probabilités d'occurrence, alors que les consommateurs plus experts prennent en compte la totalité de l'information. Il s'agirait donc d'étudier l'appréhension, la perception et le traitement du risque selon l'interaction entre l'individu et la situation, qu'il s'agisse du degré d'expertise ou de familiarité avec la situation, du modèle de choix utilisé, du degré de complexité de la tâche, du niveau d'implication, de l'occurrence de stimuli situationnels comme la publicité sur le lieu de vente etc.

A quelques exceptions près (Derbaix, 1983; Roux, 1990; Strazzieri, 1994), il est regrettable que peu d'études de chercheurs francophones ne viennent soutenir cette longue tradition de recherche. En revanche, le courant français de recherche en théorie de la décision (e.g. Munier, 1989), situé en amont de la psychologie descriptive dont nous avons principalement parlé, devrait susciter des applications plus nombreuses en psychologie du consommateur. 


\section{REFERENCES}

Abelson R.P. et Levi A. (1985), Decision Making and Decision Theory, Handbook of Social Psychology, G. Lindzey et E. Aronson Eds., Random House, NY, 231-310.

Akaah I.P. et Korgaonkar P.K. (1988), A Conjoint Investigation of the Relative Importance of Risk Relievers in Direct Marketing, Journal of Advertising Research, August, 38-44.

Alden D.L., Hoyer W.D. et Crowley A.E. (1993), Country-of-Origin, Perceived Risk and Evaluation Strategy, Advances in Consumer Research, 20, Association for Consumer Research, 678-683.

Arndt J. (1967a), Word-of-Mouth Advertising and Informal Communications, Risk Taking and Information Handling in Consumer Behavior, D.F. Cox ed., Boston, Harvard University Press, 188-239.

Arndt J. (1967b), Perceived Risk Sociometric Integration and Words-of-Mouth, Risk Taking and Information Handling in Consumer Behavior, D. Cox ed., Boston, Harvard University Press, 289316.

Athola O. (1975), The Vector Model of Preferences: An Alternative to the Fishbein Model, Journal of Marketing Research, 12, February, 52-59.

Barach J.A. (1969), Advertising Effectiveness and Risk in the Consumer Decision Process, Journal of Marketing Research, 6, August, 314-320.

Bauer R. (1960), Consumer Behavior as Risk Taking, Dynamic Marketing for a Changing World, R.S. Hancock ed., American Marketing Association, 389-398.

Bell D., Raiffa H. et Tversky A. (1988), Decision Making, Cambridge, Cambridge University Press.

Ben Zur H. et Breznitz S.J. (1981), The Effect of Time Pressure on Risky Choice Behavior, Acta Psychologica, 17, 89-104.

Bettman J.R. (1972), Perceived Risk: a Measurement Methodology and Preliminary Findings, Advances in Consumer Research, Venkatesan ed., Association for Consumer Research.

Bettman J.R. (1973), Perceived Risk and its Components: a Model and Empirical Test, Journal of Marketing Research, 10, May, 184-190.

Bettman J.R. (1979), An Information Processing Theory of Consumer Choice, Reading, MA, Addison-Wesley.

Bromiley P. et Curley S.P. (1992), Individual Differences in Risk Taking, Risk-Taking Behavior, J. Frank Yates Ed., John Wiley \& Sons, 88-132.

Chaffe S.M. et McLeod J.M. (1973), Consumer Decisions and Information use, Consumer Behavior : Theoretical Sources, S. Ward et T.S. Robertson eds.,Englewood Cliff NJ, Prentice Hall, 385-415.

Cox D.F. (1967a), Risk Handling in Consumer Behavior: Two Cases, Risk Taking and Information Handling in Consumer Behavior, D.F. Cox ed. Boston, Harvard University Press, 34-81.

Cox D.F. (1967b), Risk Taking and Information Handling in Consumer Behavior, D.F. Cox ed., Boston, Harvard University Press.

Cox D.F. (1967c), The Influence of Cognitive Needs and Styles on Information Handling in Making Product Evaluations, Risk Taking and Information Handling in Consumer Behavior, D.F. Cox ed., Boston, Harvard University Press, 370-393. 
Cox D.F. (1967d), Risk taking and Information Handling, Risk Taking and Information Handling in Consumer Behavior, D.F. Cox ed., Boston, Harvard University Press, 604-639.

Cox D.F. et Rich S.V. (1967), Perceived Risk and Consumer Decision Making - The Case of Telephone Shopping, Risk Taking and Information Handling in Consumer Behavior, D.F. Cox ed., Boston, Harvard University Press, 487-586.

Cunningham S.M. (1967a), The Majors Dimensions of Perceived Risk, Risk Taking and Information Handling in Consumer Behavior, D.F. Cox ed., Boston, Harvard University Press, 82-108.

Cunningham S.M. (1967b), Perceived Risk and Brand Loyalty, Risk Taking and Information Handling in Consumer Behavior, D.F. Cox ed., Boston, Harvard University Press, 507-523.

Currim I.S. et Sarin R.K. (1983), A Procedure for Measuring and Estimating Consumer Preferences Under Uncertainty, Journal of Marketing Research, 20, August, 249-256.

Dandouau J.C. et Bensa F. (1993), Le besoin d'information du consommateur sur le lieu de vente. Confrontation de deux échelles de mesure par ses antécédents : l'implication et le risque perçu, Cahier de Recherche, $n^{\circ}$ 9303, CREGO, IAE de Dijon, Septembre, 1-19.

Dash J., Schiffman L. et Berenson C. (1976), Risk and Personality-Related Dimensions of Store Choice, Journal of Marketing, 40, January, 32-39.

Davis J.H., Kameda T. et Stasson M.F. (1992), Group Risk Taking: Selected Topics, Risk-Taking Behavior, J. Frank Yates Ed., John Wiley \& Sons, NY, 163-199.

Derbaix C. (1983), Perceived Risk and Risk Relievers: An Empirical Investigation, Journal of Economic Psychology, 3, 19-38.

Diamond W.D. (1986), The Effect of Probability and Consequence Levels on the Focus of Consumer Judgments in Risky Situations, Journal of Consumer Research, 15, September, 280-283.

Dunn M.G., Skelly G.U. et Murphy P.E. (1986), The Influence of Perceived Risk on Brand Preference for Supermarket Products, Journal of Retailing, 62, 2, Summer, 204-216.

Engel J.F., Blackwell R.D. et Miniard P.W. (1990), Consumer Behavior, 6th Edition, NY, The Dryden Press.

Einhorn H.J. Hogarth R.M. (1986), Decision Making under Ambiguity, Journal of Business, 59, 4, S225-S250.

Festervand T.A., Snyder D.R. et Tsalikis J.D. (1986), Influence of Catalog versus Store Shopping and Prior Satisfaction on Perceived Risk, Journal of the Academy of Marketing Science, 14, 4, 28-36.

Fishbein M.A. (1967), Attitude and the Prediction of Behavior, Readings in Attitude Theory and Measurement, M. Fishbein ed., New-York, Wiley, 477-492.

Fischhoff B. (1992), Risk Taking: A Developmental Perspective, Risk-Taking Behavior, J. Frank Yates Ed., John Wiley \& Sons, NY, 133-162.

Folkes V.S. (1984), Consumer Reactions to Product Failures: an Attributional Approach, Journal of Consumer Research, 10, March, 398-409.

Folkes V.S. (1988), The Availability Heuristic and Perceived Risk, Journal of Consumer Research, 15, June, 13-23.

Forgas J.P. (1989), Mood Effects on Decision Making Strategies, Australian Journal of Psychology, 41, 197-214.

Gabbott M. (1991), The Role of Product Cues in Assessing Risk in Second-Hand Markets, European Journal of Marketing, 25, 9, 38-50.

Gijsbrechts E. (1993), Prices and Pricing Research in Consumer Marketing: Some Recent Developments, International Journal of Research in Marketing, 10, 115-151. 
Gemünden H. (1985), Perceived Risk and Information Search. A systematic Meta-Analysis of the Empirical Evidence, International Journal of Research in Marketing, 2.

Guseman D.S. (1981), Risk Perception and Risk Reduction in Consumer Services, Marketing of Services, J.A. Donelly and W.R. George eds. American Marketing Association, 200-204.

Hansen, D.E. (1992), Issues in Consumer Choice With Uncertain Product Outcomes, Advances in Consumer Research, 19, Association for Consumer Research, 175-176.

Hauser J.R. et Urban G.L. (1979), Assessment of Attribute Importances and Consumer Utility Functions: von Neumann-Morgenstern Theory Applied to Consumer Behavior, Journal of Consumer Research, 5, March, 251-262.

Havlena W.J. et DeSarbo W.S. (1991), On the Measurement of Perceived Consumer Risk, Decision Science, 22, 927-939.

Henthorne T.L., LaTour M.S. et Williams A.J. (1993), How Organizational Buyers Reduce Risk, Industrial Marketing Management, 22, 41-48.

Hisrich R., Dornoff R. et Kernan J. (1972), Perceived Risk in Store Selection, Journal of Marketing Research, 9, November, 435-439.

Hogarth R.M. (1989), Judgment and Choice: The Psychology of Decision, 2nd Edition, Chichester, John Wiley and Sons.

Hoover R., Green R. et Saegert J. A (1978), Cross-National Study of Perceived Risk, Journal of Marketing, July, 102-108.

Howard J. et Sheth N. (1969), The Theory of Buyer Behavior, NY, John Wiley \& Sons.

Humphreys M.A. et Kenderdine M. (1984), Perceived Risk and Consumer Decision Making: An Alternative View of Uncertainty, Advances in Consumer Research, 9, Association for Consumer Research, 283-285.

Ingene C.A. et Hughes M.A. (1985), Risk Management by Consumers, Research in Consumer Behavior, 1, JAI Press, 103-158.

Isen A.M. et Patrick R. (1983), The Effect of Positive Feelings on Risk Taking: When the Chips are Down, Organizational Behavior and Human Performance, 31, 194-202.

Jacoby J. et Kaplan L. (1972), The Components of Perceived Risk, Association for Consumer Research, Venkatesan M. ed., Association for Consumer Research, 287-291

Janis I.L. et Mann L. (1977), Decision Making: A Psychological Analysis of Conflict, Choice, and Commitment, Free Press, NY.

Kahn B.E. et Meyer R.J. (1991), Consumer Multiattribute Judgment under Attribute-Weight Uncertainty, Journal of Consumer Research, 17, March, 508-522.

Kahneman D. et Lovallo D. (1993), Timid Choice and Bold Forecasts: a Cognitive Perspective on Risk Taking, Management Science, 39, 1, January, 17-31.

Kahneman D. et Tversky A. (1979), Prospect Theory: an Analysis of Decision under Risk, Econometrica, 47, March, 263-291.

Kaplan L., Szybillo G.J. et Jacoby J. (1974), Components of Perceived Risk in Product Purchase: A Cross Validation, Journal of Applied Psychology, 59, 287-291.

Kogan R. et Wallach M.A. (1964), Risk Taking: A Study in Cognition and Personality, New-York, Holt, Reinhart and Winston.

Korgaonkar P. et Moschis G.P. (1989), The Effect of Perceived Risk and Social Class on Consumer Preferences for Distribution Outlets, American Marketing Association, 55, 39-43.

Knight F.H. (1921), Risk, Uncertainty, and Profit, Chicago University Press. 
Lantos G.P. (1983), The Influence of Inherent Risk and Information Acquisition on Consumer Risk Reduction Strategies, Journal of the Academy of Marketing Science, 11, Fall, 358-381.

Laurent G. et Kapferer J.N. (1986), Les profils d'implication, Recherche et Applications en Marketing, 1, 1, Avril, 41-58.

Locander W. et Hermann P. (1979), The Effect of Self-Confidence and Anxiety on Information Seeking in Consumer Risk Reduction, Journal of Marketing Research, 26, May, 268-274.

Lopes L.L. (1987), Between Hope and Fear: The Psychology of Risk, Advances in Experimental Social Psychology, 20, 255-295.

Lumpkin J.R., Crawford J.C. et Kim G. (1985), Perceived Risk as a Factor in Buying Foreign Clothes, International Journal of Advertising, 4, 157-171.

Lumpkin J.R. et Dunn M.G. (1990), Perceived Risk as a Factor in Store Choice: An Examination of Inherent Versus Handled Risk, Journal of Applied Business Research, 6, Spring, 104-118.

Lumpkin J.R. et Massey T.K. (1983), Convergent and Discriminant Validity of Alternative Measures of Perceived Risk Scales, Marketing: Theory and Concepts for an Area of Change, Summey J.H. et al. eds., Southern Marketing Association, Carbondale.

Mann L. (1992), Stress, Affect and Risk Taking, Risk-Taking Behavior, J. Frank Yates Ed., John Wiley \& Sons, NY, 200-230.

McCorkle (1990), The Role of Perceived Risk in Mail Order Catalog Shopping, Journal of Direct Marketing, 4, 4, Autumn, 26-35.

McQuarrie E.F. et Munson J.M. (1986), The Zaichkowsky Personal Involvment Inventory Modification and Extension, Advances in Consumer Research, Association for Consumer Research, 14, 36-40.

Mischel W. (1977), The Interaction of Person and Situation, Personnality at the Crossroads: Current Issues in Interactional Psychology, D. Magnusson et N.S. Endler Eds, Hillsdale, NJ, 333-352.

Mitchell V.W. (1990), Industrial Risk Reduction in The Purchase of Micro-Computers by Small Business European, Journal of Marketing, 24, 5, 7-19.

Mitchell V.W. et Boustani P. (1993), Market Development Using New Products and Customers: A Role for Perceived Risk, European Journal of Marketing, 27, 2, 17-32.

Mitchell V.W. et Greatorex M. (1989), Risk Reducing Strategies Used in the Purchase of Wine in the UK, European Journal of Marketing, 23, 9, 31-45.

Möller K.E. (1983), Perceived Uncertainty in Brand Choice: A Probabilistic Multi-Attribute Choice Model, Research in Marketing, JAI Press, 6, 199-230.

Mowen J.C. (1992), The Time and Outcome Valuation Model: Implications for Understanding Reactance and Risky Choices in Consumer Decision Making, Advances in Consumer Research, Association for Consumer Research, 19, 182-189.

Müller J. (1985), Théorie du risque perçu et comportement du consommateur : proposition d'un modèle, Thèse de 3ème cycle, Université des Sciences et Techniques, Lille, Décembre.

Muncy J.A. et Hunt S.D. (1984), Consumer Involvment: Defitional Issues and Research Directions, Advances in Consumer Research, T.C. Kinnear ed., Association for Consumer Research, 9, 193196.

Munier B. (1989), Calcul économique et révision de la théorie de la décision en avenir incertain, Revue d'Economie Politique, 99, 2, 276-306.

Murray K.B. et Schlacter J.L. (1990), The Impact of Services Versus Goods on Consumer's Assessment of Perceived Risk and Variability, Journal of the Academy of Marketing Science, 18, $1,51-65$. 
Newall J. (1977), Perceived Risk in Industrial Buying, European Journal of Marketing, 11, 173-211.

Oliver R.L. (1980), A Cognitive Model of the Antecedents and Consequences of Satisfaction Decisions, Journal of Marketing Research, 17, Novembrer, 460-469.

Perry M. et Hamm B.C. (1969), Canonical Analysis of Relations Between Socio-economic Risk and Personal Influence in Purchase Decisions, Journal of Marketing Research, 6, August, 351-354.

Peter J.P. et Ryan M. (1976), An Investigation of Perceived Risk at the Brand Level, Journal of Marketing Research, 13, May, 184-188.

Peter J.P. et Tarpey L.X. (1975), A Comparative Analysis of Three Consumer Decision Strategies, Journal of Consumer Research, 2, June, 184-189.

Pinson C., Malhotra N.K. et Jain A.K. (1984), Cognitive Differenciation in Consumer Product Judgments, Journal of Economic Psychology, 5, 353-369.

Popielarz D.T. (1967), An Exploration of Perceived Risk and Willingness to Try New Products, Journal of Marketing Research, 4, November, 368-372.

Pras B. et Summers J.O. (1978), Perceived Risk and Composition Models for Multiattribute Decisions, Journal of Marketing Research, 15, August, 429-437.

Pras B. et Tarondeau J.C. (1981), Comportement de l'acheteur, Paris, Dalloz.

Prasad V.K. (1975), Socioeconomic Product Risk and Patronage Preferences of Retail Shoppers, Journal of Marketing, 39, July, 42-47.

Puto C.P., Wesley E.P. et King R.H. (1985), Risk Handling in Industrial Vendor Selection Decisions, Journal of Marketing, 49, Winter, 89-97.

Ring A., Shriber M. et Horton R.L. (1980), Some Effects of Perceived Risk on Consumer Information Processing, Journal of the Academy of Marketing Science, 8, June, 255-263.

Rogers E.M. (1982), Diffusion of Innovations, 3rd ed., Macmillan.

Roselius T. (1971), Consumer Rankings of Risk Reduction Methods, Journal of Marketing, 36, January, 56-61.

Ross I. (1975), Perceived Risk and Consumer Behavior: a Critical Review, American Marketing Association, 1-19.

Roux E. (1991), Les facteurs explicatifs de la décision d'exporter en PMI : rôle de l'attitude du dirigeant envers le risque, Thèse d'Etat, Université de Droit, d'Economie et des Sciences d'AixMarseille III.

Schaninger C.M. (1976), Perceived Risk and Personality, Journal of Consumer Research, 3, 3, 95-100.

Shanteau J. (1992), Decision Making Under Risk: Applications to Insurance Purchasing, Advances in Consumer Research, 19, Association for Consumer Research, 177-181.

Schiffman L.G. (1972), Perceived Risk in New Product Trial by Elderly Consumers, Journal of Marketing Research, 9, 106-108.

Scheinder S.L. et Lopes L.L. (1986), Reflection in Preferences under Risk: Who and When may Suggest Why, Journal of Experimental Psychology: Human Perception and Performance, 12, 535548.

Sheth J.N. (1973), A Model of Industrial Buying Behavior, Journal of Marketing, 37, 50-56.

Shoemaker R.W. et Shoaf F.R. (1975), Behavioral Changes in the Trial of New Products, Journal of Consumer Research, 2, 2, September, 47-53.

Spence H.E., Engel J.F. et Blackwell R.D. (1970), Perceived Risk in Mail-Order and Retail Store Buying, Journal of Marketing Research, 7, August, 363-369. 
Stem D.E., Lamb C.W. et MacLachlan D.L. (1977), Perceived Risk: A Synthesis, European Journal of Marketing, 11, 4, 312-319.

Stem D.E., Lamb C.W. (1980), Segmentation of Consumer Perceptions of Risk Reduction Strategies by Product Usage and Purchase Experience, American Marketing Association, 176-179.

Stone R.N. et Gronhaug K. (1993), Perceived Risk: Further Considerations for the Marketing Discipline, European Journal of Marketing, 27, 3, 39-50.

Strazzieri A. (1992), Mesurer l'implication distinctement du risque perçu, Cahier de Recherche, $n^{\circ}$ 402, IAE d'Aix-en-Provence, Novembre, 1-19.

Strazzieri A. (1994), Mesurer l'implication durable d'un produit indépendamment du risque perçu, Recherche et Applications en marketing, 9, 1, 73-92.

Taylor J. (1974), The Role of Risk in Consumer Behavior, Journal of Marketing, 38, April, 54-60.

Thaler R. (1985), Mental Accounting and Consumer Choice, Marketing Science, 4, 199-214.

Tse D.K. et Wilton P.C. (1988), Models of Consumer Satisfaction Formation : an Extension, Journal of Marketing Research, 25, May, 204-212.

Tversky A. et Kahneman D. (1982), Judgment under Uncertainty: Heuristics and Biases, Judgment under Uncertainty, D. Kahneman ed., Cambridge, Cambridge University Press, 1-20.

Tversky A. et Kahneman D. (1992), Advances in Prospect Theory: Cumulative Representation of Uncertainty, Journal of Risk and Uncertainty, 5, 297-323.

Urbany J.E., Dickson P.R. et Wilkie W.L. (1989), Buyer Uncertainty and Information Search, Journal of Consumer Research, 16, September, 208-215.

Vann J.W. (1983), A Multi-distributional Conceptual Framework for the Study of Perceived Risk, Advances in Consumer Research, Association for Consumer Research, 10, 442-446.

Vann J.W. (1987), A Conditional Probability View of the Role of Product Warranties in Reducing Perceived Financial Risk, Advances in Consumer Research, Association for Consumer Research, 14, 421-425.

Venkatraman, M.P. (1989), Involvment and Risk, Psychology and Marketing, 6, 3, 229-247.

Verhage B.J., Yavas U. et Green R.T. (1990), Perceived Risk: A Cross-Cultural Phenomenon?, International Journal of Research in Marketing, 7, 297-303.

Von Neumann J. et Morgenstern O. (1947), Theory of Games and Economic Behavior.

Woodruff R.B. (1972), Brand Information Sources Opinion Change and Uncertainty, Journal of Marketing Research, 9, November, 414-418.

Woodside A. (1972), Informal Group Influence on Risk Taking, Journal of Marketing Research, 9, May, 223-225.

Woodside A.G. et DeLozier M.W. (1976), Effects of Word-of-Mouth Advertising on Consumer RiskTaking, Journal of Advertising, 5, Fall, 12-19.

Yates J.F. et Stone E.R. (1992a), The Risk Construct, Risk-Taking Behavior, J. Frank Yates Ed., John Wiley \& Sons, NY, 1-25.

Yates J.F. et Stone E.R. (1992b), Risk Appraisal, Risk-Taking Behavior, J. Frank Yates Ed., John Wiley \& Sons, NY, 49-86.

Zikmund W.G. et Scott I.E. (1973), A Multivariate Analysis of Perceived Risk Self-Confidence and Information Source, Advances in Consumer Research, 1, Association for Consumer Research.

Zeithaml V.A. (1988), Consumer Perceptions of Price, Quality, and Value: A Means-End Model and Synthesis of Evidence, Journal of Marketing, 52, July, 2-22. 
Figure 1 - Schéma intégrateur

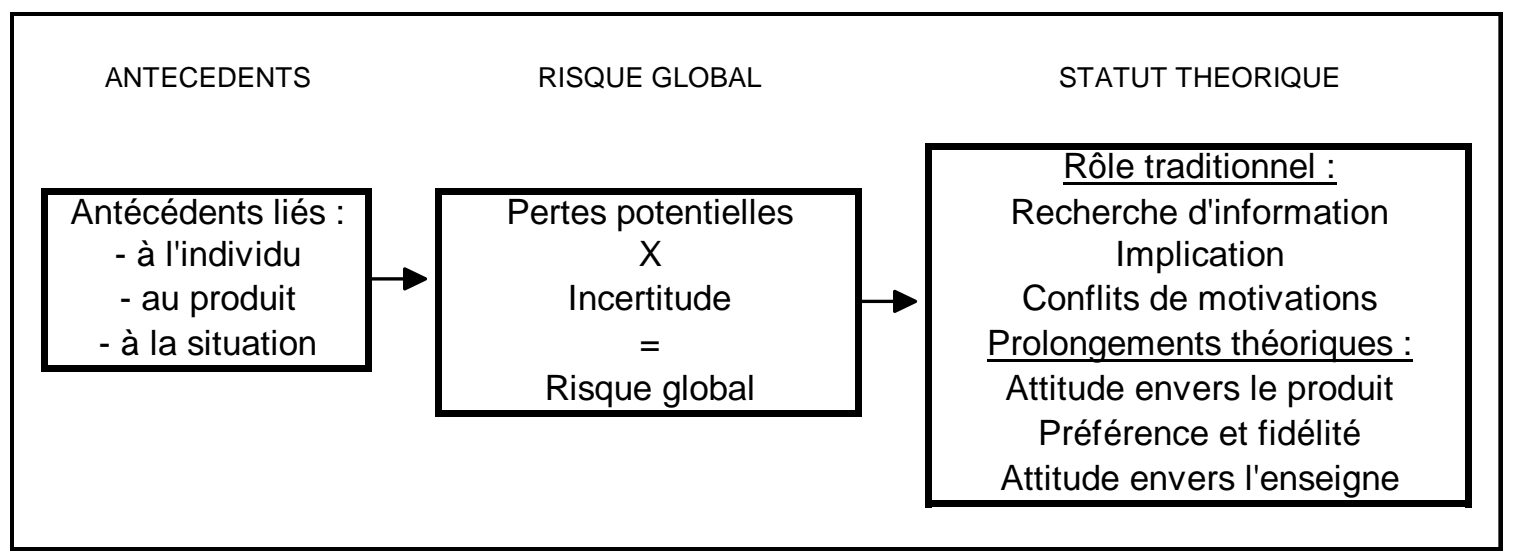

Figure 2 - Les composantes du risque perçu

EVALUATION DES PERTES POTENTIELLES

- Identification des alternatives
- Identification et estimation des attributs
- Estimation des points de référence
- Evaluation des attributs

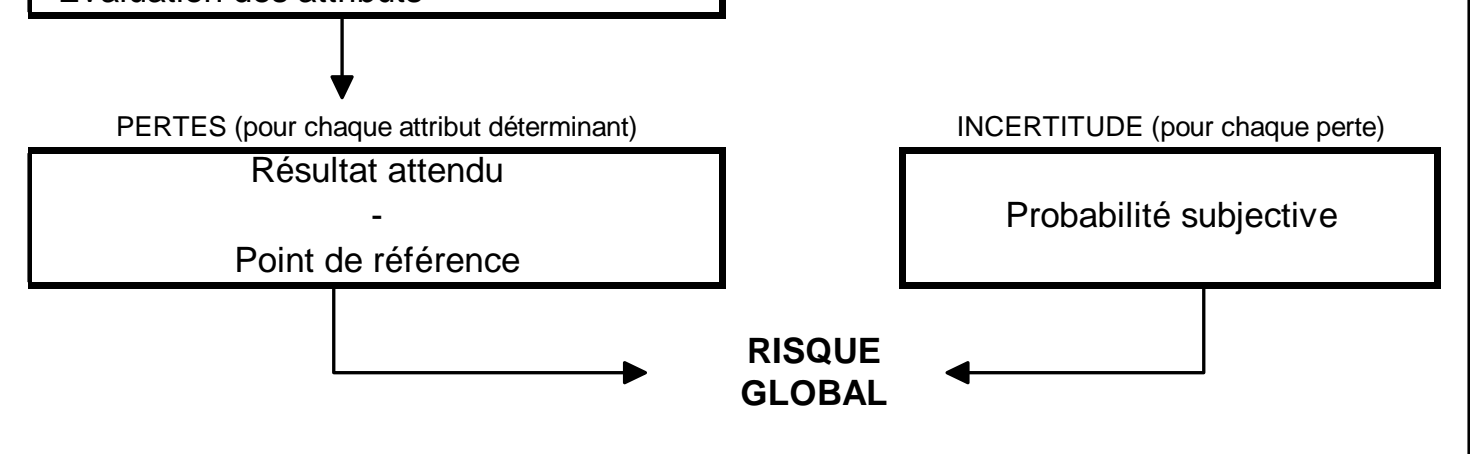


Figure 3 - Les antécédents du risque perçu

\begin{tabular}{|c|c|c|}
\hline \multirow{3}{*}{$\begin{array}{l}\text { - Confiance en soi } \\
\text { - Style cognitif } \\
\text { - Anxiété } \\
\text { - Attitude face au risque } \\
\text { - Variables socio- } \\
\text { démographiques } \\
\text { - Culture (pays) } \\
\text { - Motivation } \\
\text { - Implication durable } \\
\end{array}$} & PRODUIT & \multirow{3}{*}{\begin{tabular}{l}
\multicolumn{1}{c}{ SITUATION } \\
- Cadrage et biais cognitifs \\
- Emotions \\
- Temps \\
- Conflits \\
- Mode ou lieu d'achat \\
- Définition de la tâche \\
- Activité au point de vente \\
--- variables non traitées ---- \\
- Situation d'achat industriel \\
- Influence d'un groupe
\end{tabular}} \\
\hline & $\begin{array}{l}\text { - Classe de produit } \\
\text { - Pays d'origine } \\
\text { - Marque } \\
\text { - Prix } \\
\text { - Nature (bien ou service) }\end{array}$ & \\
\hline & & \\
\hline
\end{tabular}

Figure 4 - Le statut théorique du risque perçu

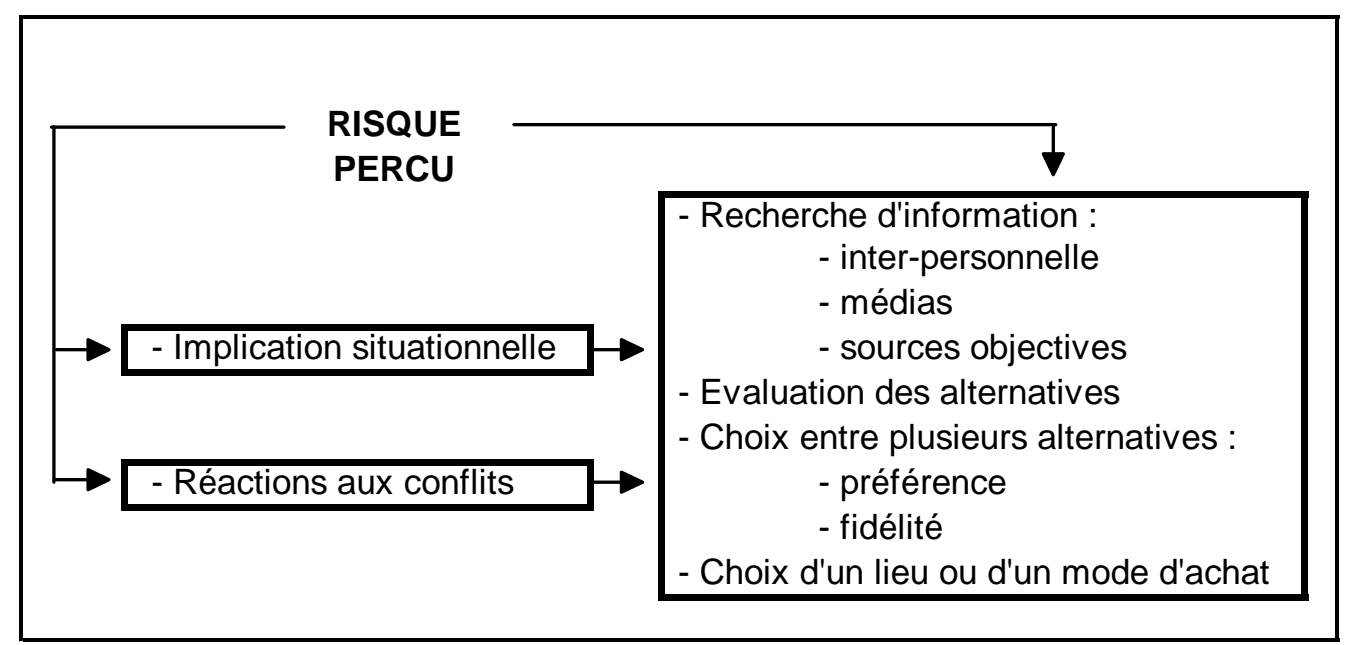


Tableau 1 - Etapes du processus d'évaluation de l'incertitude

\begin{tabular}{|l|l|}
\hline Identification des alternatives & $\begin{array}{l}\text { Quels modèles examiner : Peugeot 106, Citroën } \\
\text { AX, Renault Clio, Volkswagen Polo, Ford Fiesta... ? }\end{array}$ \\
\hline $\begin{array}{l}\text { Identification des attributs } \\
\text { importants dans le choix }\end{array}$ & $\begin{array}{l}\text { Prix, garantie, délai de réparation, réputation du } \\
\text { constructeur, systèmes de financement, tests de } \\
\text { sécurité...? }\end{array}$ \\
\hline Estimation des attributs & $\begin{array}{l}\text { Quel est le prix de la Clio, la durée de garantie de la } \\
106, \text { le degré de sécurité à conduire une Fiesta... ? }\end{array}$ \\
\hline Estimation des points de référence & $\begin{array}{l}\text { Budget (prix maximal), exigences de sécurité (air } \\
\text { bag, système de freinage ABS...), durée de garantie } \\
\text { souhaitable...? }\end{array}$ \\
\hline Evaluation des attributs & $\begin{array}{l}\text { Le prix d'une Fiesta est inacceptable pour mon } \\
\text { budget, la sécurité d'une Clio est bonne et meilleure } \\
\text { que..., la garantie Peugeot } 106 \text { est correcte mais } \\
\text { moins longue que... }\end{array}$ \\
\hline
\end{tabular}

Tableau 2 - Réduction d'une liste d'attributs en facteurs de risque d'après Havlena et DeSarbo (1991)

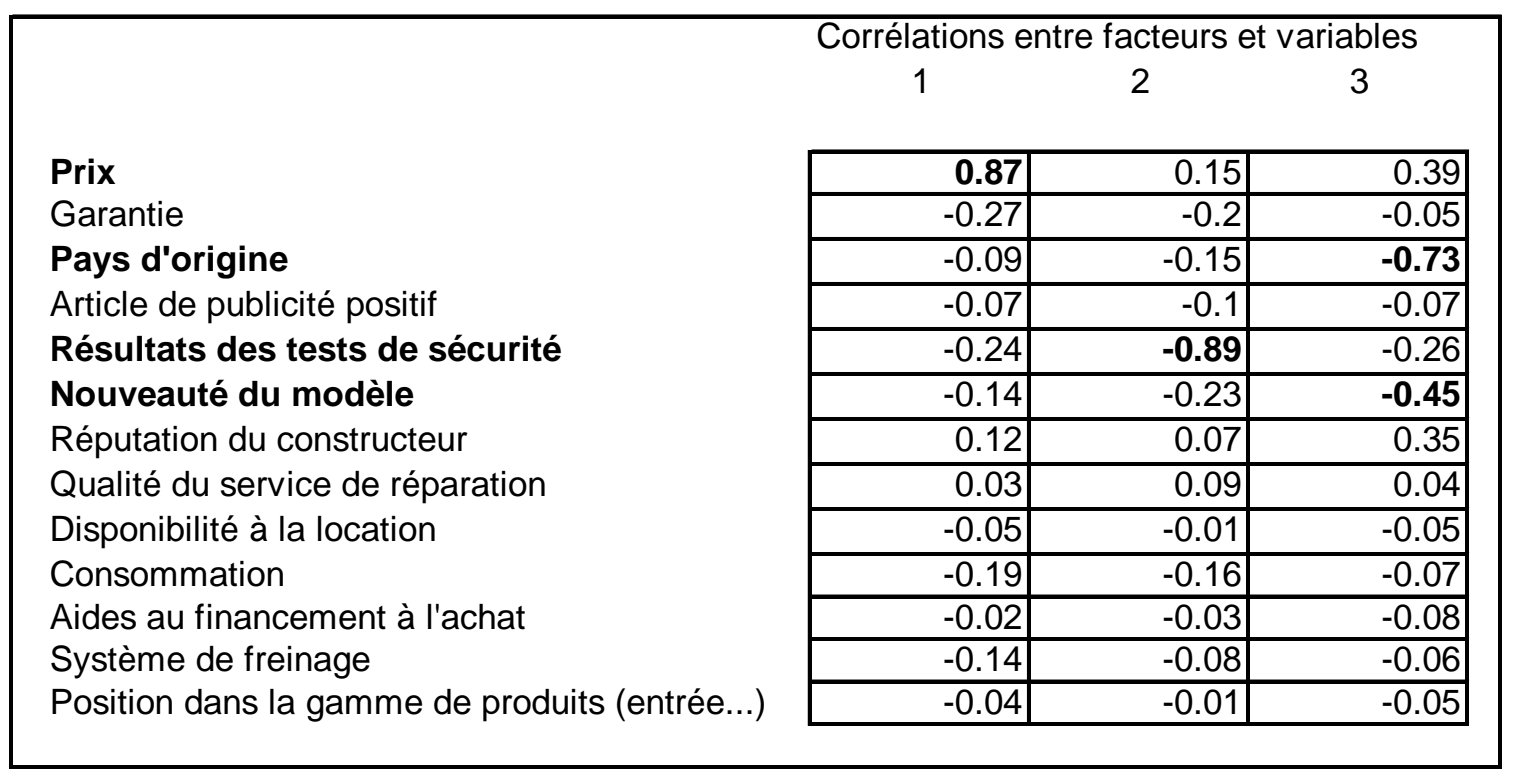


Tableau 3 - Mesure du risque perçu par la méthode de Cunningham (1967)

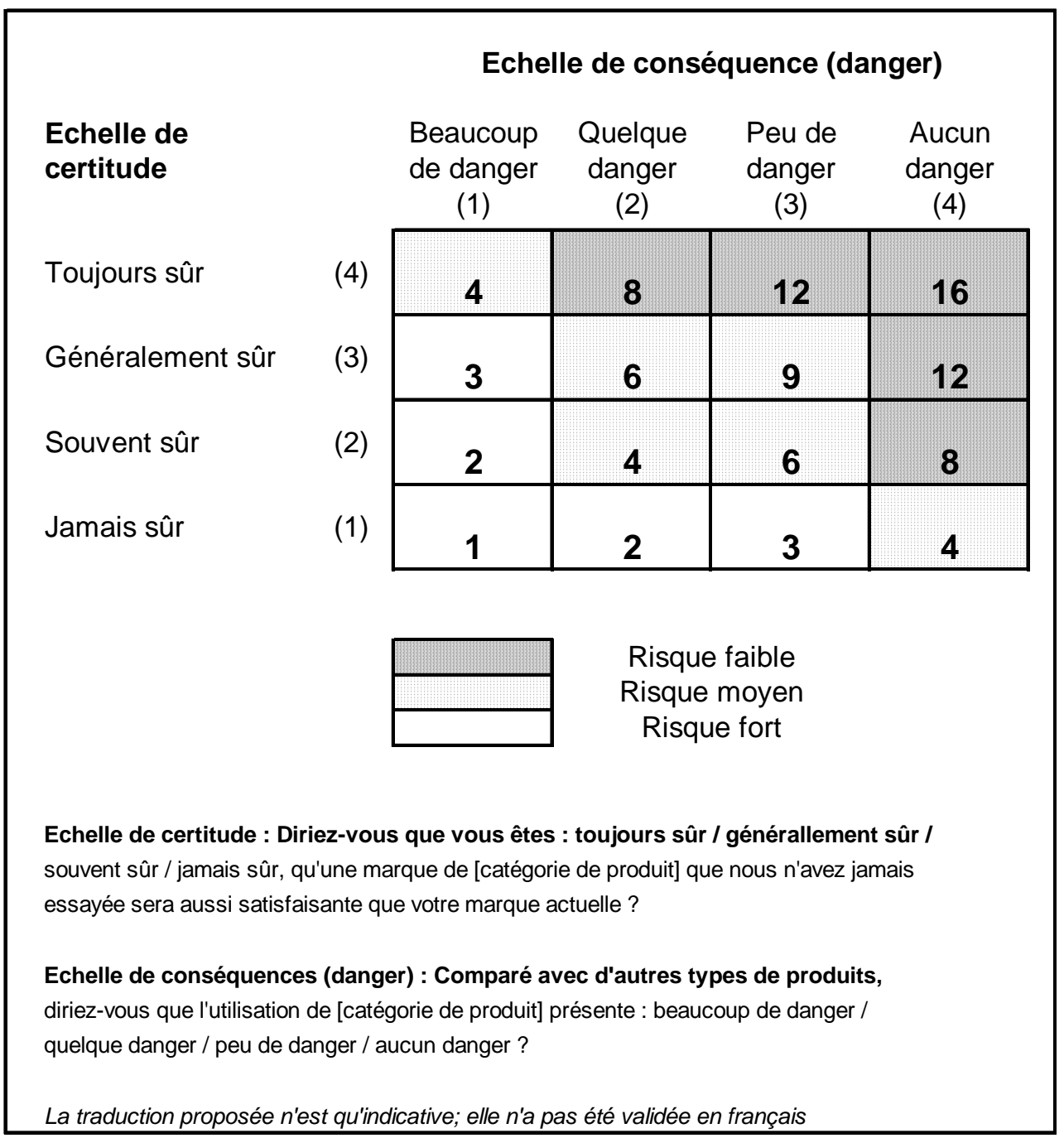

